\title{
Epigenetic control: slow and global, nimble and local
}

\author{
Christine S. Cheng, ${ }^{1}$ Tracy L. Johnson, ${ }^{2}$ and Alexander Hoffmann ${ }^{1,3}$ \\ ${ }^{1}$ Signaling Systems Laboratory, Department of Chemistry and Biochemistry, University of California, San Diego, \\ La Jolla, California 92093, USA; ${ }^{2}$ Division of Biology, University of California, San Diego, La Jolla, California 92093, USA
}

The regulation of gene expression involves multiple levels of control, from those that are inheritable to those that are highly responsive to environmental changes. In this issue of Genes \& Development, Dong and colleagues (pp. 1159-1173) demonstrate that the dynamically controlled immune response transcription factor NF-кB may, in fact, have a role in regulating heterochromatin and gene expression at large distances from its actual target sequences and genes.

How the cell regulates the use of its genetic information has been a primary focus of molecular biological research from its inception more than 50 years ago. Two general mechanisms have framed our understanding of how and when the information contained within the DNA is "read" by the cell: those that impede access to the DNA sequence via nucleosome-mediated packaging or DNA methylation, and those mediated by sequence-specific DNA-binding proteins that recruit transcriptional machinery to promoters to activate gene expression. The genome organization into discrete euchromatic and heterochromatic regions (particularly at centromeres, telomeres, and other discrete loci) suggested early on that this packaging was relatively stable and could thereby be inherited to play a key role in development. On the other hand, sequence-specific binding proteins were thought to mediate more nimble, gene-specific control in response to rapidly changing cellular environments. Over the past decade, post-translational modifications of histone tails have emerged as the central regulation step in both types of control, operating at different spatial/temporal scales: the "global" scale that regulates portions of a chromosome at sub-cell cycle time scales, and the "local" scale regulating single transcription units to satisfy dynamically changing physiological needs. However, in this issue of Genes \& Development, Dong et al. (2008) provide evidence that suggests a provocative connection between a dynamically regulated transcription factor ca-

[Keywords: Epigenetics; inflammation; NF-кB; gene expression; phosphorylation]

${ }^{3}$ Corresponding author.

E-MAIL ahoffmann@ucsd.edu; FAX (858) 822-4671.

Article is online at http://www.genesdev.org/cgi/doi/10.1101/gad.1677008. pable of activating specific target genes, and nonlocal control of gene repression.

\section{Heterochromatin: global control}

The term "epigenetics" was coined by Conrad Waddington (Waddington 1942) to account for mechanisms by which genetic information is selectively used as cells differentiate into more specialized functions. The concept of an epigenetic landscape is predicated on the idea that repressive chromatin compaction leads to deselection of genetic information. This pattern is inherited, leading to progressive restriction of cell fate and resulting in differentiation and development. The characteristic chromatin compaction pattern of Drosophila interphase salivary gland chromosomes-where euchromatic and heterochromatic regions are easily distinguished in the microscope-provided an early visual correlate to the accessible and inaccessible genetic loci. This type of epigenetic regulation encompasses multiple genes, is cell type-specific, and relatively stable-less stable than genetic information itself, but more stable than the cellular regulation demanded by physiological responses, metabolic changes, or pathogen infection.

The stability of epigenetic memory emerged from the study of remarkable phenomena in several model systems (for a recent review, see Grewal and Jia 2007). The mating-type locus in yeast is regulated by heterochromatin formation and maintenance (Grunstein 1998); in Schizosaccharomyces pombe it is stable through $>2000$ generations, providing a fine example of epigenetic inheritance. As recently explored with a simple three-state mathematical model for chromatin regulation, this stability depends on cooperative interactions of the repressive state (Dodd et al. 2007). Indeed, the second hallmark of heterochromatin is that it propagates in a sequenceindependent manner (Fig. 1A). In other words, epigenetic silencing is nonlocal and does not depend on sequencespecific DNA-binding proteins. In fact, sequence elements are more likely involved in limiting the spread of heterochromatin (Gaszner and Felsenfeld 2006) such as those that flank the mating-type loci. DNA-binding factors that are recruited to such boundary-defining elements might interrupt the assembly of the nucleosomal 
A.

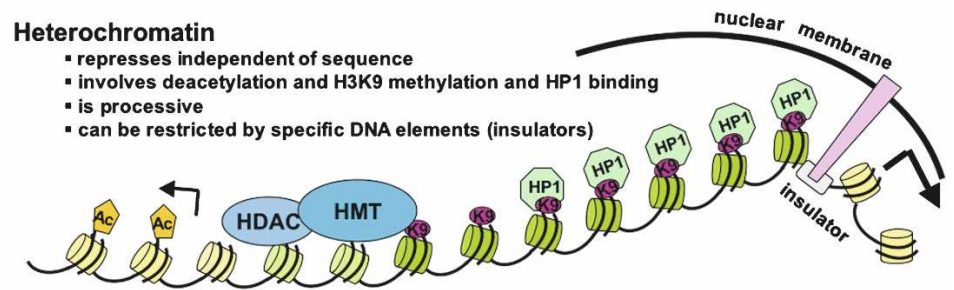

B.

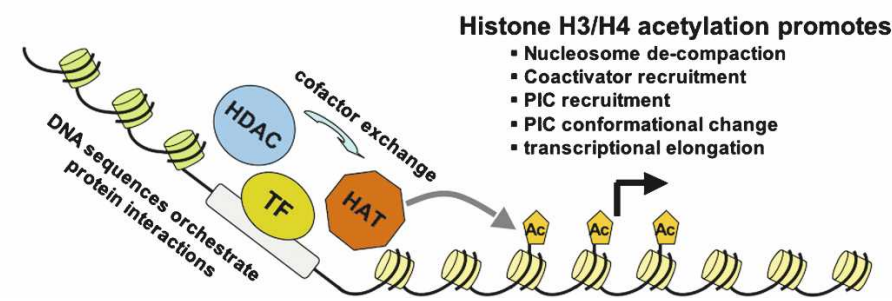

Figure 1. Long-range heterochromatin repression and local transcriptional activation. (A) Establishment of heterochromatin involves removal of acetyl groups from histone tails (by HDAC), methylation of Lys 9 on histone $\mathrm{H} 3$ (H3K9me; by histone methyltransferase), and recruitment of chromodomain-containing proteins such as HP1. Heterochromatin is inherently processive, with the underlying cooperativity also accounting for its stability. Heterochromatin spreading can be blocked by transcriptionally active regions or insulators that may be associated with nuclear membrane and pore complexes. Nucleation and maintenance do not require sequencespecific DNA-binding proteins but are mediated by repetitive DNA sequences, RNAi machinery, or noncoding scaffold RNAs (such as Xist or HOTAIR). Some nuclear envelope/nuclear pore components appear to function as insulators. $(B)$ Stimulusinduced transcriptional activation of a specific gene involves transcription factors whose recruitment is orchestrated by enhancers and promoters that contain clustered binding sites. In addition, these DNAbinding factors may need to be modified to allow for an exchange of corepressors (such as HDAC) with coactivators (such as HATs). Local acetylation of the histones of a single or a few nucleosomes may effect gene activation by affecting nucleosome compaction, recruiting additional coactivators, or components of the PIC, such as TAF1, which contains acetyl-lysine-binding bromodomains, or effecting conformational changes in a previously assembled PIC to allow for RNA Pol II recruitment, initiation, or release.

array, which appears to be critical for heterochromatin spreading, or they might recruit histone modifiers that counteract the molecular characteristics of heterochromatin (Zofall and Grewal 2006). The role of heterochromatin spreading was powerfully revealed in Drosophila mutants, where inadvertent spread of gene silencing from neighboring regions affects the expression of genes regulating eye color. Suppressor screens of such heterochromatin misregulation revealed some of the factors controlling heterochromatin regulation.

At the same time, an increasing set of analytical tools allowed characterization of post-translational modifications of histone tails. Methylation of Lys 9 on histone H3 ( $\mathrm{H} 3 \mathrm{~K} 9 \mathrm{me})$ is commonly associated with heterochromatin, leading to the recruitment of chromodomain proteins such as HP1 (Maison and Almouzni 2004). Insulator elements and cognate binding factors, such as the mammalian CTCF, as well as recently discovered scaffold RNAs (Rinn et al. 2007), play an important role in heterochromatin initiation, definition, and maintenance, apparently in conjunction with nuclear membrane-anchored scaffolds (Ishii et al. 2002) and the threedimensional organization of chromatin within the nucleus (Akhtar and Gasser 2007).

\section{Transcription factors: local control}

Quite in parallel but often separately, gene-specific regulation of expression has been investigated. These studies were kicked off by the identification of sequence elements that direct or activate transcriptional initiation (Benoist and Chambon 1981). Specific DNA-binding transcription factors were identified and shown to mediate a promoter code in activating transcription in a highly gene-specific manner (Ptashne and Gann 1990), while biochemical studies identified components of the core promoter machinery (Roeder 1991) that forms the preinitiation complex (PIC) and orchestrates the mechanisms underlying initiation by RNA polymerase II (Pol II) (Fig. 1B).

How do transcriptional activators binding to specific promoter sequences activate transcription? Analogous to bacterial gene control, recruitment of the core machinery, especially components of the TFIID complex, preoccupied research in the early 1990s (Ptashne and Gann 1997), but other models including conformational changes in TFIID (Horikoshi et al. 1988; Hoffmann et al. 1997) or nucleosomal rearrangement (Workman et al. 1988) were also investigated.

Although some early data indicated that mutants in histone tails can have gene-specific transcriptional regulation defects (Grunstein 1990), the degree to which histone tail modification would dominate the discussion of the transcriptional activation mechanism over the past decade is remarkable. When the sequence-specific DNAbinding transcription factor's elusive "targets" (Ptashne and Gann 1990) or coactivators were identified not merely as recruitment adaptors but enzymes capable of adding or removing histone tail modifications, it became clear that local nucleosomal control, or local epigenetics, is intimately involved in local gene regulation. Indeed, while the word epigenetics suggested DNA and nucleosomal modification to encode hereditary information, the current paradigm of gene regulation holds that at least some nucleosomal modifications are nimble and reversible enough that they can function to regulate transcription locally and gene-specifically via a proposed code of histone tail modifications (Jenuwein and Allis 2001). 


\section{NF-кB in epigenetic control}

The transcription factor NF- $\mathrm{BB}$ is known for being highly dynamically regulated (Hoffmann et al. 2002). Although the protein factor itself is constitutively present, its activity is inducible via stimulus-induced degradation of inhibitor proteins (the IкBs), some of which provide negative feedback (Hoffmann and Baltimore 2006). Indeed, the NF- $\mathrm{kB}$ signaling module is capable of generating stimulus-specific dynamic control of NF- $\mathrm{B}$ activity (Werner et al. 2005). Such temporally defined control is reflected in NF-кB's primary function in orchestrating inflammatory and immune responses in both innate and adaptive compartments. Although NF-кB's primary activating component RelA contains one of the most potent transcriptional activation domains, it remains somewhat unclear how NF-кB actually regulates transcription.

One of the most important transcriptional activation mechanisms appears to involve the histone acetyltransferase (HAT) CBP/p300 (Perkins et al. 1997), a widely recognized coactivator present in limiting amounts in the cell. There are two domains within $\mathrm{CBP} / \mathrm{p} 300$ capable of binding the NF-кB subunit RelA when it is phosphorylated at Ser 276 (Zhong et al. 1998). That phosphorylation event occurs during NF- $\mathrm{B}$ activation in response to stimulation with cytokines or pathogen-derived substances and was first described in the laboratory of Sankar Ghosh (Zhong et al. 1997).

In parallel, other studies have focused on the small but detectable amount of constitutive or basal nuclear DNAbinding activity of NF- $\mathrm{B}$, which may be linked to an inactive gene expression state. Indeed, nonphosphorylated Ser 276-containing RelA was found to be associated with histone deacetylases (HDACs) (Zhong et al. 2002; Chen and Greene 2004). These studies suggested that in addition to IкB-mediated regulation of nuclear localization, NF- $\kappa B^{\prime}$ s transcriptional effect may also be controlled by recruitment and exchange of corepressors and coactivators, akin to the well-documented regulation of nuclear hormone receptor transcription factors (Rosenfeld et al. 2006).

In this issue of Genes \& Development, the laboratory of Sankar Ghosh (Dong et al. 2008) report a knock-in mutant mouse strain containing the nonphosphorylateable RelA S276A variant, which allowed them to separate the NF- $\kappa$ B control mechanisms of IкB-mediated nuclear localization and of regulated transcriptional cofactor recruitment. As predicted, some but not all NF-кB-responsive gene expression was found to be attenuated in the mutant cells, as they are presumably dependent on CBP recruitment and histone acetylation marks. However, quite unexpectedly, the mutation also produced a variety of embryonic developmental defects, most obvious in eye development, that varied markedly among individuals, similar to the variegated Drosophila eye color mutants caused by misregulated heterochromatic silencing. Eye development is normal in NF-кB knockout embryos (including rela $\mathrm{a}^{-/}$) (Hoffmann and Baltimore 2006), and the known developmental genes (such as Pax6) are not among the many known NF- $\mathrm{B}$ response genes whose expression can be induced by NF-кB-inducing stimuli (http://www.nf-kb.org). Hence, the finding suggests a provocative connection between locally bound sequence-specific DNA-binding transcriptional regulators, and nonlocal control of gene repression.

\section{Local events with global effects}

Why would a highly dynamically regulated, sequencespecific DNA-binding transcription factor participate in long-term and nonlocal regulation of DNA accessibility and chromatin packaging? Barring alternate interpretations of the mutant knock-in mouse experiments presented by Dong et al. (2008), we may consider how or why such a regulatory connection may regulate human physiology. At its minimum, the limitations imposed on heterochromatin spreading may simply be imperfect, as distances between genes are not always large, allowing local control to spill over to neighbors. Such sloppy regulation may be accentuated in a mutant that is locked in the "off" position and that may act as a dominant negative by constitutively recruiting HDACs. In that scenario, it remains to be investigated whether basal NF$\kappa \mathrm{B}$-independent transcription, basal NF- $\kappa \mathrm{B}$ activity, or occasional bursts of NF- $\mathrm{kB}$ activity are required to ward off chromatin spreading.

However, chances are that epigenetic regulation is intricately coordinated across all time scales and distances such that wild-type NF-кB may, in fact, have a role in heterochromatin regulation. Epigenetic regulation maintains homeostatic states that not only produce constitutive gene expression, but that also allow for nimble regulation of individual genes by determining the "poised" gene regulation states. Such epigenetic states are regulated on a large scale to guide cellular differentiation during development and, interestingly, they may be affected by transient gene expression activities. Even transiently active transcription factors-in response to extracellular signals, for example-may not only reversibly alter local chromatin modification, but may also leave a cellular memory of their activation in terms of an altered balance in chromatin marks.

In the case of NF- $\kappa \mathrm{B}$, transient activation of NF- $\kappa \mathrm{B}$ via the inflammatory response canonical signaling pathway, for example, leaves a cellular memory for immune developmental noncanonical signaling in regulating lymph node development and homeostasis (Basak et al. 2007). It remains to be seen, then, what the role of epigenomic vestiges of transient activation of NF- $\mathrm{kB}$ during immune or inflammatory responses might be, for example, in adaptive immune cell development or the formation of immune memory or tolerance.

There are, indeed, other recent indications that sequence-specific DNA-binding factors are able to initiate heterochromatic silencing (Grewal and Jia 2007). The stress-activated transcription factor ATF/CREB is recruited to a specific CRE element at the mating-type region of $S$. pombe to initiate heterochromatin nucleation. Interestingly, ATF recruits HDACs to nucleate 
and maintain the heterochromatin by stabilizing the H3K9 methylation marks and HP1 recruitment, and to restrict the accessibility of the basal transcription machinery including Pol II (Yamada et al. 2005). Similarly, the cell cycle transcriptional repressor Rb, a well-known tumor suppressor, is required for the recruitment of SUV39H1 (which methylates H3K9) and HP1 to repress genes that are required for the entry into $S$ phase (such as Cyclin E and cyclin A2) (Nielsen et al. 2001). Interestingly, this kind of repression is transient, although it uses the same molecular characteristics of heterochromatin, such as H3K9 methylation marks and HP1.

Indeed, two recent developments indicate that heterochromatin regulation in mammals may not be as large scale or as permanent as early work in model organisms first suggested. Although methyl modifications of lysines are chemically stable, more than half a dozen enzymes have been discovered that demethylate Lys 9 of histone $\mathrm{H} 3$, thus rendering the hallmark modification of heterochromatin reversible (Kouzarides 2007). Expression of a demethylase that reverses the (Polycomb protein-mediated) repression-associated methyl mark $\mathrm{H} 3 \mathrm{~K} 27 \mathrm{me} 3$ is highly inducible, in fact, in response to NF- $\mathrm{BB}$ activity (De Santa et al. 2007), thereby allowing for trans-differentiation of macrophages in response to inducing signals within inflamed tissues and challenging Waddinton's early concept of the epigenetic landscape. Furthermore, in the chromatin contained in nuclei of differentiated cells, cell fate-restricting chromatin marks can be erased when placed into appropriate cytoplasmic contexts (Hochedlinger and Jaenisch 2006) or, in most recent work, when a combination of master regulator transcription factors is provided (Jaenisch and Young 2008) to generate totipotent embryonic stem cells.

Together, it appears that the layers of gene expression, although functioning on distinct scales of time and distance, are intricately linked via histone modifications. Epigenetic regulation controls DNA accessibility over multigenic regions, but while these epigenic states can be inherited and restrict the possible gene expression programs in a given cell, they can also be reversed, even within post-mitotic cells, by recruiting enzymes that erase relevant repressive marks. Dong et al. (2008) provide evidence for the converse - that a transcription factor associated with the transient regulation of specific genes also affects the homeostatic regulation of chromatin accessibility over larger regions. As in so many other examples of biology, understanding the mechanisms of gene regulation requires consideration of disparate mechanisms in an intricate network. That sort of functional cross-talk will surely be reflected in increasingly close interactions between previously distinct communities of researchers.

\section{References}

Akhtar, A. and Gasser, S.M. 2007. The nuclear envelope and transcriptional control. Nat. Rev. Genet. 8: 507-517.

Basak, S., Kim, H., Kearns, J.D., Tergaonkar, V., O'Dea, E., Werner, S.L., Benedict, C.A., Ware, C.F., Ghosh, G., Verma,
I.M., et al. 2007. A fourth IкB protein within the NF-кB signaling module. Cell 128: 369-381.

Benoist, C. and Chambon, P. 1981. In vivo sequence requirements of the SV40 early promotor region. Nature 290: 304 310.

Chen, L.F. and Greene, W.C. 2004. Shaping the nuclear action of NF-кB. Nat. Rev. Mol. Cell Biol. 5: 392-401.

De Santa, F., Totaro, M.G., Prosperini, E., Notarbartolo, S., Testa, G., and Natoli, G. 2007. The histone H3 lysine-27 demethylase Jmjd3 links inflammation to inhibition of polycomb-mediated gene silencing. Cell 130: 1083-1094.

Dodd, I.B., Micheelsen, M.A., Sneppen, K., and Thon, G. 2007. Theoretical analysis of epigenetic cell memory by nucleosome modification. Cell 129: 813-822.

Dong, J., Jimi, E., Zhong, H., Hayden, M.S., and Ghosh, S. 2008. Repression of gene expression by unphopshorylated NF-кB p65 through epigenetic mechanisms. Genes \& Dev. (this issue). doi: $10.1101 / \mathrm{gad} .1657408$.

Gaszner, M. and Felsenfeld, G. 2006. Insulators: Exploiting transcriptional and epigenetic mechanisms. Nat. Rev. Genet. 7: 703-713.

Grewal, S.I. and Jia, S. 2007. Heterochromatin revisited. Nat. Rev. Genet. 8: 35-46.

Grunstein, M. 1990. Nucleosomes: Regulators of transcription. Trends Genet. 6: 395-400.

Grunstein, M. 1998. Yeast heterochromatin: Regulation of its assembly and inheritance by histones. Cell 93: 325-328.

Hochedlinger, K. and Jaenisch, R. 2006. Nuclear reprogramming and pluripotency. Nature 441: 1061-1067.

Hoffmann, A. and Baltimore, D. 2006. Circuitry of nuclear factor $\mathrm{\kappa B}$ signaling. Immunol. Rev. 210: 171-186.

Hoffmann, A., Oelgeschlager, T., and Roeder, R.G. 1997. Considerations of transcriptional control mechanisms: Do TFIID-core promoter complexes recapitulate nucleosome-like functions? Proc. Natl. Acad. Sci. 94: 89288935.

Hoffmann, A., Levchenko, A., Scott, M.L., and Baltimore, D. 2002. The ІкB-NF-кB signaling module: Temporal control and selective gene activation. Science 298: 1241-1245.

Horikoshi, M., Hai, T., Lin, Y.S., Green, M.R., and Roeder, R.G. 1988. Transcription factor ATF interacts with the TATA factor to facilitate establishment of a preinitiation complex. Cell 54: 1033-1042.

Ishii, K., Arib, G., Lin, C., Van Houwe, G., and Laemmli, U.K. 2002. Chromatin boundaries in budding yeast: The nuclear pore connection. Cell 109: 551-562.

Jaenisch, R. and Young, R. 2008. Stem cells, the molecular circuitry of pluripotency and nuclear reprogramming. Cell 132: 567-582.

Jenuwein, T. and Allis, C.D. 2001. Translating the histone code. Science 293: 1074-1080.

Kouzarides, T. 2007. Chromatin modifications and their function. Cell 128: 693-705.

Maison, C. and Almouzni, G. 2004. HP1 and the dynamics of heterochromatin maintenance. Nat. Rev. Mol. Cell Biol. 5: 296-304.

Nielsen, S.J., Schneider, R., Bauer, U.M., Bannister, A.J., Morrison, A., O'Carroll, D., Firestein, R., Cleary, M., Jenuwein, T., Herrera, R.E., et al. 2001. Rb targets histone H3 methylation and HP1 to promoters. Nature 412: 561-565.

Perkins, N.D., Felzien, L.K., Betts, J.C., Leung, K., Beach, D.H., and Nabel, G.J. 1997. Regulation of NF-кB by cyclin-dependent kinases associated with the p300 coactivator. Science 275: 523-527.

Ptashne, M. and Gann, A.A. 1990. Activators and targets. Nature 346: 329-331. 
Cheng et al.

Ptashne, M. and Gann, A. 1997. Transcriptional activation by recruitment. Nature 386: 569-577.

Rinn, J.L., Kertesz, M., Wang, J.K., Squazzo, S.L., Xu, X., Brugmann, S.A., Goodnough, L.H., Helms, J.A., Farnham, P.J., Segal, E., et al. 2007. Functional demarcation of active and silent chromatin domains in human HOX loci by noncoding RNAs. Cell 129: 1311-1323.

Roeder, R.G. 1991. The complexities of eukaryotic transcription initiation: Regulation of preinitiation complex assembly. Trends Biochem. Sci. 16: 402-408.

Rosenfeld, M.G., Lunyak, V.V., and Glass, C.K. 2006. Sensors and signals: A coactivator/corepressor/epigenetic code for integrating signal-dependent programs of transcriptional response. Genes \& Dev. 20: 1405-1428.

Waddington, C. 1942. The epigenotype. Endeavour 1: 18-20.

Werner, S.L., Barken, D., and Hoffmann, A. 2005. Stimulus specificity of gene expression programs determined by temporal control of IKK activity. Science 309: 1857-1861.

Workman, J.L., Abmayr, S.M., Cromlish, W.A., and Roeder, R.G. 1988. Transcriptional regulation by the immediate early protein of pseudorabies virus during in vitro nucleosome assembly. Cell 55: 211-219.

Yamada, T., Fischle, W., Sugiyama, T., Allis, C.D., and Grewal, S.I. 2005. The nucleation and maintenance of heterochromatin by a histone deacetylase in fission yeast. Mol. Cell 20: 173-185.

Zhong, H., SuYang, H., Erdjument-Bromage, H., Tempst, P., and Ghosh, S. 1997. The transcriptional activity of NF-кB is regulated by the IкB-associated PKAc subunit through a cyclic AMP-independent mechanism. Cell 89: 413-424.

Zhong, H., Voll, R.E., and Ghosh, S. 1998. Phosphorylation of NFкB p65 by PKA stimulates transcriptional activity by promoting a novel bivalent interaction with the coactivator CBP/p300. Mol. Cell 1: 661-671.

Zhong, H., May, M.J., Jimi, E., and Ghosh, S. 2002. The phosphorylation status of nuclear NF- $\mathrm{KB}$ determines its association with CBP/p300 or HDAC-1. Mol. Cell 9: 625-636.

Zofall, M. and Grewal, S.I. 2006. Swi6/HP1 recruits a JmjC domain protein to facilitate transcription of heterochromatic repeats. Mol. Cell 22: 681-692. 


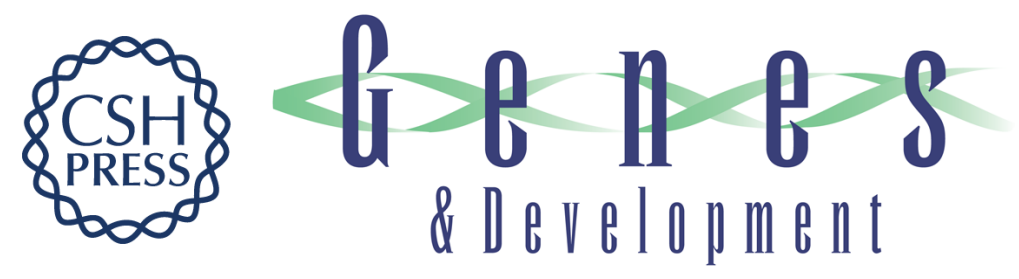

\section{Epigenetic control: slow and global, nimble and local}

Christine S. Cheng, Tracy L. Johnson and Alexander Hoffmann

Genes Dev. 2008, 22:

Access the most recent version at doi:10.1101/gad.1677008

Related Content Repression of gene expression by unphosphorylated NF-B p65 through epigenetic mechanisms Jie Dong, Eijiro Jimi, Haihong Zhong, et al.

Genes Dev. May , 2008 22: 1159-1173

References This article cites 36 articles, 7 of which can be accessed free at: http://genesdev.cshlp.org/content/22/9/1110.full.html\#ref-list-1

Articles cited in:

http://genesdev.cshlp.org/content/22/9/1110.full.html\#related-urls

\section{License}

Email Alerting

Receive free email alerts when new articles cite this article - sign up in the box at the top

Service right corner of the article or click here.

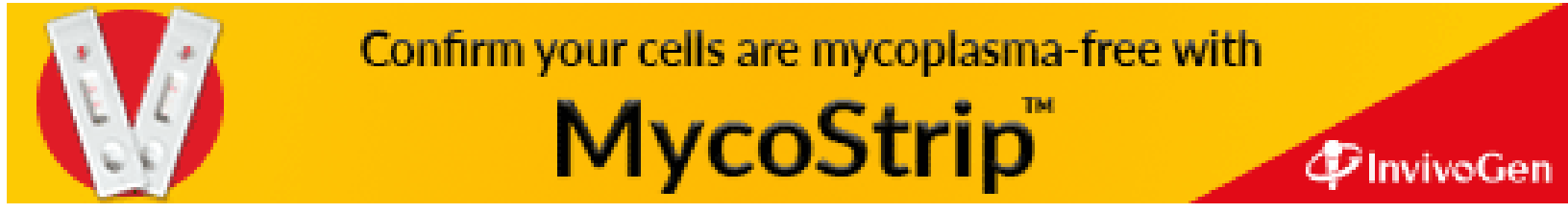

